\title{
Telematikeinsatz im Baustellenbetrieb zur optimalen Transportabwicklung
}

\author{
Prof. DR.-ING. DIPL.-WIRTSCH.-ING. W.A. GÜNTHNER, \\ DIPL.-ING. S. KESSLER, \\ DIPL.-ING. S. SANLADERER \\ LEHRSTUHL FÜR FÖRDERTECHNIK MATERIALFLUSS UND LOGISTIK, TU MÜNCHEN
}

Dem Thema Baustellenlogistik wurde sowohl in der Baubranche selbst als auch in der Forschung in den letzten Jahren nur begrenzte Aufmerksamkeit gewidmet. Durch die Optimierung der baunahen Prozesse, wie beispielsweise Transport von Stück- und Schüttgütern, lassen sich noch weit reichende Einsparpotenziale realisieren. Im Rahmen eines AiF $^{1}$ Forschungsprojektes wurde am Lehrstuhl für Fördertechnik Materialfluss Logistik (fml) der TU München in Zusammenarbeit mit Industriepartnern ein Flottentelematiksystem für die Baubranche unter Berücksichtigung der besonderen Randbedingungen, entwickelt und in einem langfristigen Pilotversuch getestet. Ein zentraler Bestandteil des Systems stellt die Anbindung an ein übergeordnetes Back Office System (SAP) dar, an das alle relevanten Informationen übermittelt werden und somit für die Abrechung und statistische Auswertungen zur Verfügung stehen.

Both the construction industry and the research dedicated only limited attention to the subject of construction-site logistics during the past years, even though great potential savings can be realized by optimizing construction related processes, e.g., the transport of unit load and bulk load. In the scope of an Aif $^{2}$ research project and in cooperation with industrial partners the institute for materials handling material flow and logistics (Fördertechnik Materialfluss Logistik - fml) of the Technical University of Munich, developed a fleet telematik system for the construction industry. It takes into account the industry's special boundary conditions and has been tested in a long-term test run. A central module of the system is the connection to a back office system (SAP). All relevant information is transmitted to this system and is available for clearing and statistical reporting.

Die deutsche Bauindustrie sieht sich in den kommenden Jahren mit einer Vielzahl an zusätzlichen Herausforderungen konfrontiert, die sie im Gegensatz zu den Wettbewerbern besonders aus Billiglohnländern meistern muss. Hierzu zählen besonders die stark gestiegenen Energie- und Rohstoffkosten, aber auch der langfristig schrumpfende innerdeutsche Absatz für Bauleistungen. Eine Studie der Deutschen Bank Research zeigt deutlich, dass auch in den kommenden Jahren keine signifikante Besserung der Auftragslage in der Baubranche zu erwarten ist, obwohl sich das derzeitige Investitionsvolumen bereits seit einigen Jahren auf sehr niedrigem Niveau mit einem permanenten leichten Abwärtstrend bewegt. Mittelfristig werden die Investitionen auf Grund bestehender Überkapazitäten speziell im Industriellen Hochbau auf diesem niedrigen Niveau stagnieren. Zusätzlich zeichnet sich im Wohnungsbau in Deutschland, bedingt durch einen Bestandes der großteils älter als 50 Jahre ist, ein Trend hin zu mehr Sanierungsarbeiten an bestehenden Bauobjekten ab. Dadurch müssen die Firmen mit geänderten Leistungsanforderungen speziell in der Bauplanung und der Baustellenlogistik rechnen und ihre Arbeitsabläufe kostenoptimal anpassen.

Langfristig wird die Nachfrage an Bauleistungen im Hoch- und Wohnungsbau im Bezug auf das heutige Investitionsvolumen sogar noch abnehmen, da durch die demographische Bevölkerungsentwicklung im Zeitraum bis 2050 weniger Bedarf an Wohn- und Industriebauten entsteht. Der Bereich des Tiefbaus stellt in dieser Entwicklung noch eine Ausnahme zu diesem Trend dar, da einerseits großer Nachholbedarf durch mangelnde Investitionen im Infrastrukturbereich der letzten Jahre besteht, andererseits müssen zur Bewältigung des steigenden Verkehrsaufkommens durch die zentrale Position Deutschlands in einem vereinten Europa mittelund langfristig große Neu- und vor allem Sanierungsinvestitionen in die gesamte Verkehrsinfrastruktur getätigt werden. Positiv für die Investitionen im Bereich der Infrastruktur werden sich auch die Einnahmen durch die LKW-Maut bemerkbar machen, die auch künftig ein gleich bleibend hohes Investitionsvolumen sichern können.

\footnotetext{
${ }^{1}$ Arbeitsgemeinschaft industrieller Forschungsvereinigungen „Otto-von-Guericke“ e.V. - AiF

${ }^{2}$ German Federation of Industrial Research Associations "Otto von Guericke" - AiF
} 

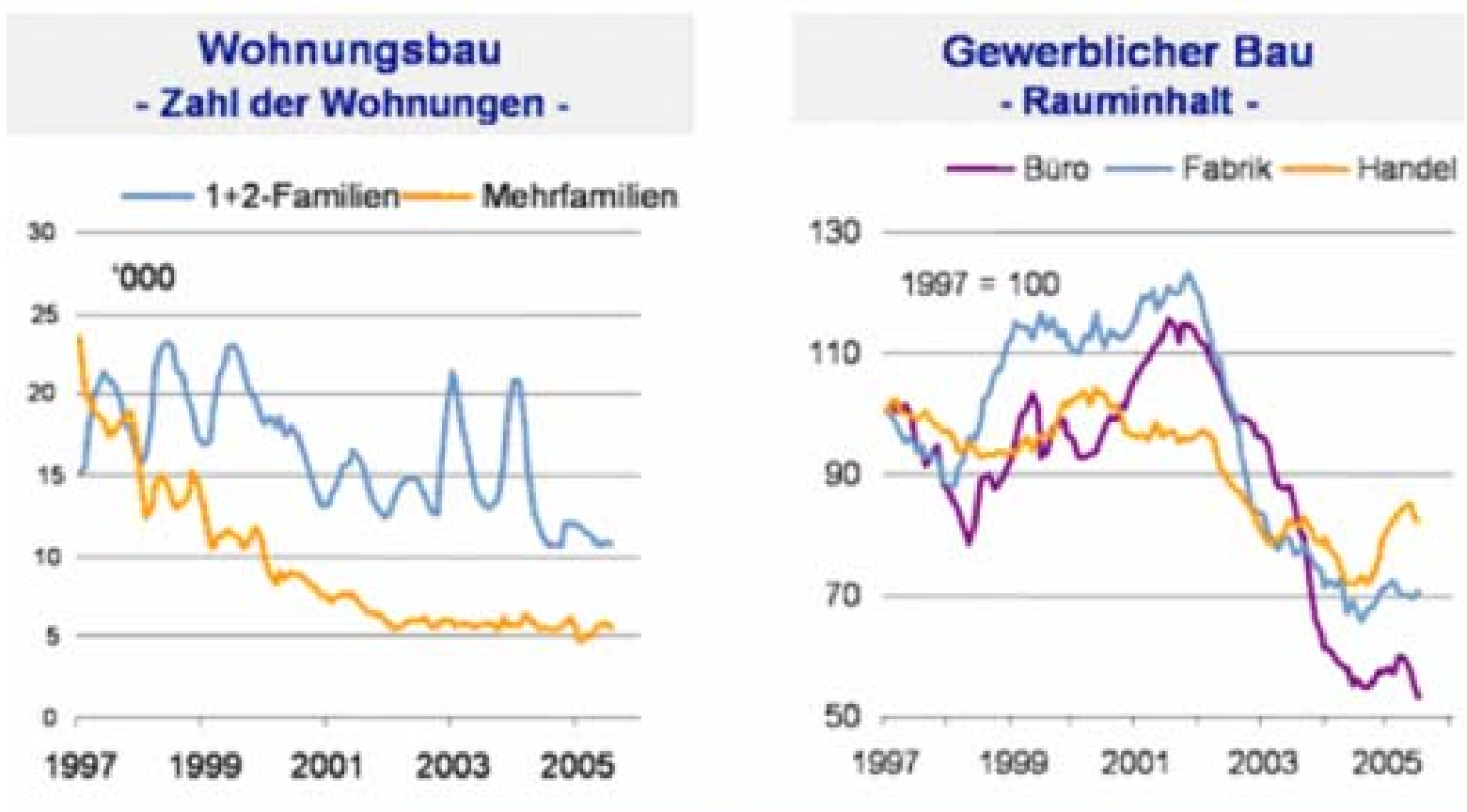

1992-1999 2000-2004 2005-2009

\begin{tabular}{|l|c|c|c|}
\hline Wohnungsbau & 3,5 & $-4,0$ & 0,5 \\
\hline Sonst. Hochbau & $-1,0$ & $-4,1$ & $-0,3$ \\
\hline Tiefbau & 0,5 & $-1,9$ & 0,5 \\
\hline Bau insgesamt & 1,7 & $-3,4$ & 0,5 \\
\hline
\end{tabular}

Abbildung 1: Prognose für die Entwicklung der deutschen Bauwirtschaft , reale durchschnittliche Veränderung in \% zum vorigen Zeitraum,

[Quellen: Statistisches . BundesamtA, DB Research (2005 und 2006)]

Für Bauunternehmen stellt sich daher die Aufgabe, ihre Prozesse sowohl in der physischen Bauabwicklung, als auch in der Planung sowie im Bereich Abrechnung und Controlling, kritisch zu überprüfen und wenn nötig anzupassen, um in den stark umkämpften Märkten wie auch in neuen Marktnischen Aufträge zu erhalten und diese gewinnorientiert abwickeln zu können. Somit kann auch in einer harten Wettbewerbssituation der Unternehmenserfolg dauerhaft gesichert werden.

\section{Logistikoptimierung als Wettbewerbsvorteil}

Da der Logistik in der Baubranche lange Zeit nur geringe Bedeutung beigemessen wurde, sind auf diesem Gebiet noch besonders hohe Verbesserungs- und Einsparungspotenziale vorhanden. Vor allem Bereiche außerhalb der eigentlichen Kernkompetenzen der technischen Bauabwicklung, wie beispielsweise der Maschinen- und Fuhrpark, sind lohnenswerte Objekte für Kosteneinsparungsprojekte. 
Die Transporte zu und auf den Baustellen stellen zwar für die Praxis eine Notwendigkeit dar und sie bilden einen relativ großen Kostenfaktor des gesamten Bauprozesses, diese Dienstleistung ist aber für das gesamte Bauwerk und im Normalfall auch für den Bauherren nur von untergeordneter Bedeutung, da sie im Endergebnis nicht mehr sichtbar ist. Es gilt daher diese nicht wertschöpfenden Tätigkeiten einerseits mit minimalen Kosten, andererseits aber mit hoher Qualität durchzuführen.

Eine Option zur Reduzierung der Kosten im Bereich Fuhrpark ist das Outsourcing der Fahrzeuge und der gesamten Verwaltung. Damit sind jedoch auch Nachteile, wie geringere Flexibilität und Abhängigkeit von einem Dienstleister sowie geringer Einfluss auf die Qualität der erbrachten Leistung, verbunden. Eine geeignete Alternative stellt eine Optimierung der Prozesse innerhalb des eigenen Fuhrparks durch den Einsatz von Telematik-/ EDV-Systemen für die Planung und Abwicklung der Transporte dar. Für eine derartige EDV Unterstützung ist allerdings eine Analyse der organisatorischen Aufgaben im Vorfeld unerlässlich, um die Software individuell an die firmenspezifischen Prozesse anpassen zu können.

\section{Schwachstellen in der Fuhrpark-Abwicklung}

Das Tagesgeschäft ist sehr stark geprägt von kurzfristigen Ablaufänderungen, bedingt durch nicht vorhersehbare Witterungseinflüsse oder unerwartete Bauverzögerungen. Da die faktischen Transportaufträge auf der Baustelle meist nicht bekannt sind, ist für die Fahrzeuge somit keine Disposition im eigentlichen Sinne und auch kein objektives, zeitnahes Controlling der LKW möglich. Eine direkte Steuerung der Fahrzeuge während des Tages, um die Transportaufträge optimal zu verteilen, ist zudem nur bedingt realisierbar. Fahrzeuge werden üblicherweise für einen bestimmten Zeitraum einer Baustelle zugewiesen und dort direkt vom Polier den Aufgaben zugeteilt, ohne dass der Disponent eine genaue Rückmeldung über den Einsatz seiner Fahrzeuge erhält. Eine genaue Kontrolle der Leistung innerhalb der Baustelle ist nur sehr eingeschränkt durchführbar und hängt stark von der Genauigkeit der Leistungsdokumentation auf den LKW-Tagesberichten ab, da diese von jedem Fahrer manuell angefertigt und anschließend zur Abrechnung der erbrachten Leistungen in der Verwaltung per Hand in einem EDV-System erfasst und ausgewertet werden. Trotz dieses erheblichen Aufwands ist die Aktualität der Auswertungen nicht besonders hoch, da eine manuelle Datenerfassung für viele Fahrzeuge sehr zeitintensiv ist und auch der Rücklauf der Berichte, besonders bei weit entfernten Baustellen, nur diskontinuierlich erfolgt. Die manuelle Datenerfassung einer Vielzahl an Berichten und Lieferscheinen ist einerseits mit hohen Personalkosten verbunden, andererseits stellt diese Tätigkeit eine zusätzliche „Fehlerquelle“ dar. Leistungsdaten sind somit bei größeren Fuhrparks nicht mit vertretbarem Aufwand tagesaktuell zu verarbeiten und zu überprüfen.

Zusätzlich wird die Fahrzeugdisposition durch die Tatsache erschwert, dass die Beschaffung von Informationen über eine Vielzahl von Transportfahrzeugen mit hohem Zeit- und Kostenaufwand verbunden ist, da aktuelle Informationen meist über Handy abgefragt werden müssen. Baustellentransporte müssen aber auf Grund des steigenden Kostendrucks immer öfter Just in Time (JIT) mit möglichst geringen Standzeiten und optimierten Fahrzeugauslastungen durchgeführt werden. Besonders bei qualitativ anspruchsvollen und zeitkritischen Baumaßnahmen, wie beispielsweise dem Einbau von Feste-Fahrbahn-Plattenelementen für den ICE, die individuell für die genaue Einbauposition auf eine Genauigkeit von wenigen Hundertstel Millimeter geschliffen werden, ist zusätzlich die produktionssynchrone Anlieferung in der richtigen Fertigungsreihenfolge (Just in Sequence - JIS) eine entscheidende Anforderung an die Transportaufgabe, die der Disponent erfüllen muss. Für eine JIT bzw. JIS-Anlieferung müssen dem Disponenten daher jederzeit genaue Informationen sowohl über den Standort und Status eines Fahrzeugs als auch im Fall der Stückguttransporte über die Ladung selbst zur Verfügung stehen. Derzeit ist diese Vielzahl an Informationen dem Disponenten nur mit hohem Aufwand zugänglich, da sie in verschiedenen Systemen zwar generiert werden, aber je nach Informationswunsch manuell kombiniert werden müssen. 


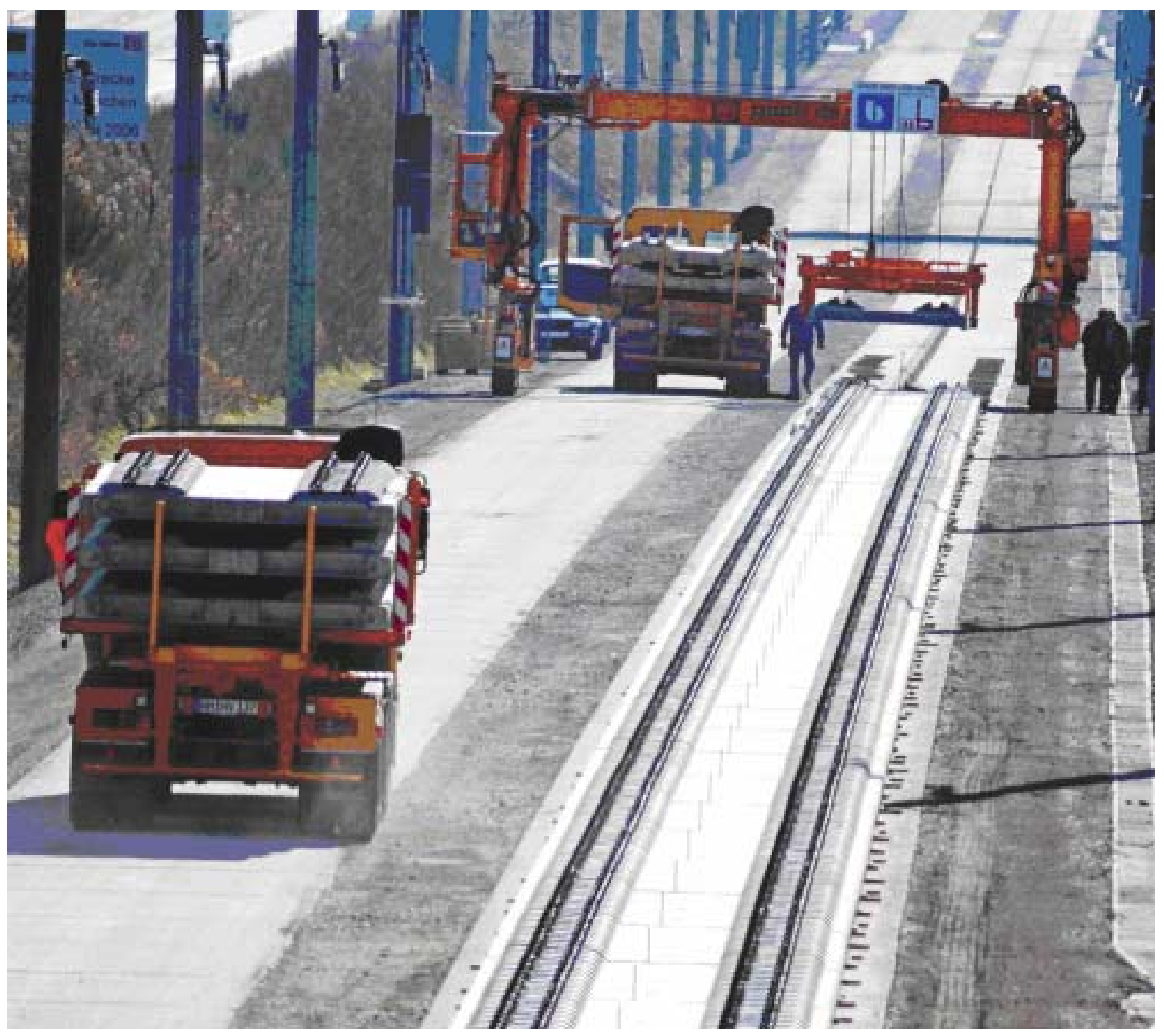

Abbildung 2: JIS Anlieferung beim Einbau von Feste Fahrbahn Elementen

Wichtig bei der Transportlogistik am Bau ist die Integration in den Gesamtprozess der Fertigung unter besonderer Berücksichtung der Themen Auftragsabwicklung, Qualitätssicherung sowie der Abstimmung der vorhandenen Kapazitäten mit den Wünschen der Kunden (Baustellen) gemäß Anlieferungszeitpunkt, Flexibilität, Lieferservice etc.

Zur Umsetzung dieser Ziele werden in den Fuhrparkabteilungen vieler Firmen laufend Verbesserungen der Dispositionsarbeit durch die Mitarbeiter selbst durchgeführt, die vor allem auf Lerneffekten und Optimierungen der Arbeit durch individuelle Hilfsmittel basieren. Die Auswirkungen dieser evolutionären Verbesserungsschritte sind jedoch begrenzt und hängen stark von der persönlichen Arbeitsweise jedes einzelnen Mitarbeiters ab. Problematisch ist auch die Vielzahl an unterschiedlichen Vorgehensweisen und die oft schlechte Nachverfolgbarkeit der zu erledigenden Aufgaben, falls kurzfristig ein Mitarbeiter ausfällt.

Um weitere grundsätzliche Verbesserungen in der Prozessabwicklung erzielen zu können, ist es für innovative Unternehmen immer wieder nötig, das Gesamtsystem und dessen Abwicklung genau zu analysieren und die eingefahrenen internen Abläufe zu überdenken. Unter Berücksichtigung der technischen Entwicklungen und einem sinnvollen Einsatz neuer Medien kann somit in vielen Fällen ein „Evolutionssprung“ erreicht werden, der neue Optimierungspotenziale erschließt und die Unternehmen wettbewerbsfähig für neue Aufgaben macht.

Im Falle der Transport und Geräte GmbH (TuG) der Firma Max Bögl markierte diesen Schritt die Einführung eines Fuhrparktelematiksystems zur Steuerung der 250 Fahrzeuge, der in Zusammenarbeit mit dem Lehrstuhl fml der Technischen Universität München (TUM) durch ein gemeinsames Forschungsprojekt im Herbst des Jahres 2003 initiiert wurde. Dieses Forschungsprojekt baut auf den Erkenntnissen eines AIFForschungsvorhabens auf, in dem ein Demonstrator ähnlicher Konzeption für den Transport veredelter Schüttgüter (z. B. Splitte, Asphalt und Transportbeton) entwickelt wurde. Letzteres Projekt wurde im Auftrag der Bundesvereinigung Logistik (BVL) e.V. durchgeführt und aus Mitteln des Bundesministeriums für Wirtschaft über die Arbeitsgemeinschaft industrieller Forschungsvereinigungen "Otto von Guericke" e.V. (AiF) gefördert. 


\section{Aufgaben der Telematik zur Fuhrparksteuerung bei der Fa. Max Bögl}

Ziel des Telematikeinsatzes ist die Optimierung der gesamten Prozesskette von den Produktionswerken bis hin zur Belieferung der Baustellen mit Schüttgütern, wie Asphalt-Mischgut, Transportbeton sowie Betonfertigteilen. Neben der reinen Fahrzeugdisposition sind die beiden weiteren Hauptziele des Transportlogistik-Projektes die durchgängige Integration der Lohn- und Leistungsabrechnung sowie die Erfassung von Qualitäts- und Leistungskennwerten für die Kunden (siehe Abb. 3). Durch die Eingliederung in die bestehende SAPLandschaft, können am Bordrechner erfasste Daten automatisch verarbeitet und manuelle Arbeitsschritte zur Abrechnung so weit als möglich vermieden werden. Somit reduziert sich der Zeitbedarf und Arbeitsaufwand zur gesamten Auftragsabwicklung erheblich.

Zeitnahe statistische und abrechnungsrelevante Auswertungen sowohl für die Kunden, als auch für interne Stellen können mit geringem Aufwand automatisiert zur Verfügung gestellt werden. So können einerseits die Transportkosten durch den optimierten Einsatz der Fahrzeuge gesenkt und andererseits eine lückenlose digitale Qualitätsdokumentation gewährleistet werden. Zusätzlich können aus den protokollierten Daten der Fahrzeuge Leistungskennwerte generiert und dem Kunden für statistische Zwecke zur Verfügung gestellt werden. Weiterhin soll die Planungssicherheit und Transparenz der externen sowie internen Transporte auf der Baustelle verbessert werden. Dies betrifft einerseits die Zusammenarbeit mit den Baustellen und Werken im Vorfeld der Planung, um die Transporte optimal abwickeln zu können, andererseits auch die Kontrolle der Fahrzeuge während des Tages durch eine Onlineübertragung von Statusdaten, die dem Disponenten jederzeit Auskunft über die Tätigkeit seiner LKW liefern.

Der Fuhrpark wandelt sich somit zu einem integrierten Dienstleister, der durch die Bereitstellung von qualitativ hochwertigen Daten hinsichtlich Leistung und Aufwand einen Mehrwert sowohl für die Baustelle, als auch für die Lieferwerke generiert, da alle Beteiligten besser in den Material- und Informationsfluss integriert werden.

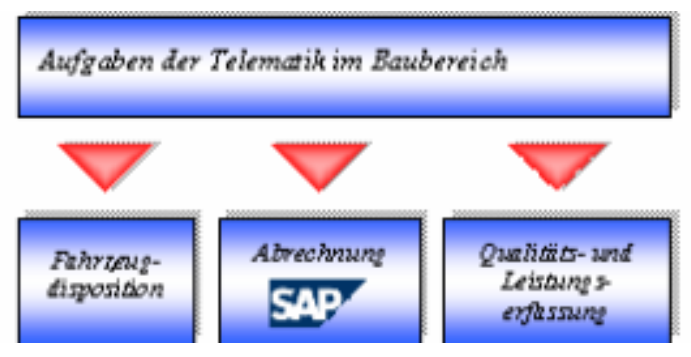

Abbildung 3: Einsatzfelder der Telematik

Diese Kernziele des Einsatzes von Telematik in der Baubranche lassen sich mit den am Markt verfügbaren Systemen, die hauptsächlich auf den klassischen Speditionsbetrieb mit fester Routenplanung und längeren Fahrstrecken zugeschnitten sind, nur bedingt abbilden. Die erste Aufgabe im Projekt bestand daher in der Definition der erforderlichen Prozesse, um die unterschiedlichen Transportarten im Baugewerbe, wie Gerätetransport, Schüttguttransport zur Werksversorgung, interne Baustellentransporte etc. bestmöglich mit EDV-Unterstützung abbilden zu können. Ein wichtiges Entscheidungskriterium für die Auswahl der Hard- und Software war die einfache intuitive Handhabung, um sowohl den Disponenten als auch den Fahrern ein Arbeitshilfsmittel an die Hand zu geben, das die Arbeitsprozesse bestmöglich unterstützt und somit auf breite Akzeptanz stößt.

\section{Hardware}

Die verwendete Hardware besteht aus einem Bordrechner, der an die Fahrzeugelektronik zur Erfassung der gefahrenen Kilometer und der Kippvorgänge angeschlossen wird, und einem Touchscreen-Display zur Bedienung (siehe Abb. 4) durch den Fahrer. Der Rechner ist universell einsetzbar und kann im Gegensatz zu den herstellerbezogenen Insellösungen für Fahrzeugtelematiksysteme in die LKW verschiedener Hersteller problemlos eingebaut werden. Für gemischte Flotten kann somit ein einheitliches Gerät eingesetzt werden, bei dem alle Fahrer unabhängig vom aktuellen Fahrzeug immer mit demselben System arbeiten können. 
Bei der Auswahl der Geräte wurde besondere Rücksicht auf eine bedienerfreundliche sowie robuste Ausführung geachtet, da die Geräte durch den Einsatz speziell innerhalb der Baustelle harten Umgebungsbedingungen, vor allem durch die Vibrationen und Verschmutzung ausgesetzt sind.

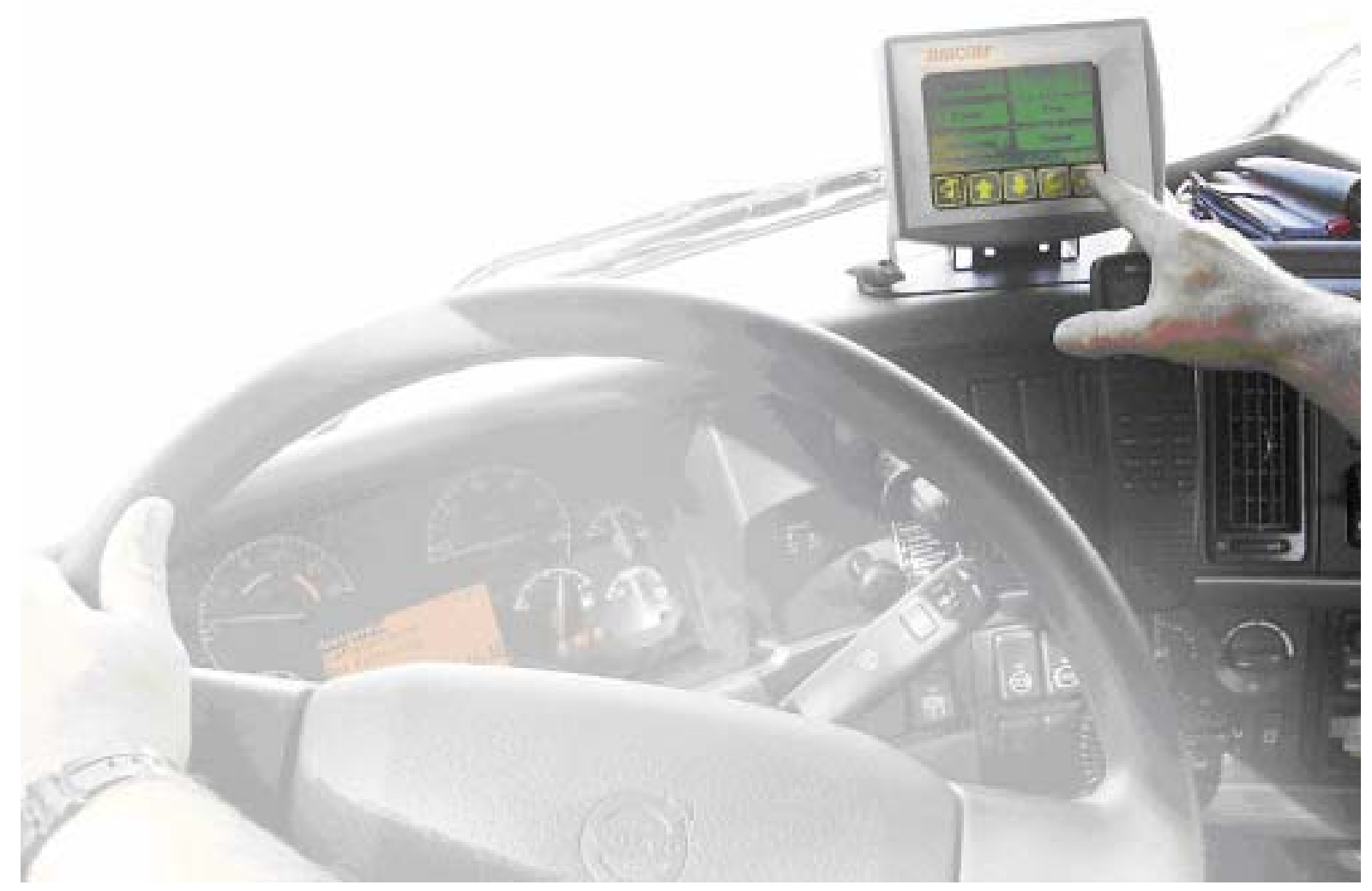

Abbildung 4: Einbaubeispiel Bordrechner [Foto Max Bögl]

Der Fahrer hat mittels vordefinierter Meldungen am Display die Möglichkeit, seine aktuelle Tätigkeit, wie beispielsweise Reparatur/Wartung oder auch Pause, an die Zentrale zu übermitteln, damit der Disponent jederzeit die Zustände seiner Fahrzeuge nachvollziehen kann. Weiterhin können auch individuelle Meldungen ähnlich einer SMS (Short Message Service) verfasst werden, um mit dem Disponenten in Kontakt zu treten.

Die Kommunikation mit den Bordrechnern erfolgt über GPRS (General Packet Radio Service), da eine Echtzeitübertragung der Daten gefordert wurde. Für diesen Einsatzfall bietet GPRS im Vergleich zu einer Datenübertragung per SMS deutliche Kostenvorteile, da beim datenbasierten GPRS nicht die Anzahl der Verbindungen, sondern lediglich die übertragenen Datenmengen Kosten verursachen. Da das Datenvolumen einer Meldung durch die Codierung sehr gering ist (ca. 160 bis 200 Byte pro Meldung), entstehen zu der Grundgebühr des m2m-Tarifes (machine to machine Tarif der Telekom mit Flatrate) keine zusätzlichen Mobilfunk-Gebühren. Mittels eines GPS-Empfängers wird permanent die Fahrzeugposition bestimmt, so dass bei jeder Statusmeldung der aktuelle Standort an das zentrale System übermittelt werden kann.

Zur Gewährleistung der Übertragungssicherheit verfügen die Bordrechner über einen Sicherheits-Puffer, der bei fehlender Funkverbindung alle Status- und Auftragsmeldungen zwischenspeichert und alle bis dahin aufgelaufenen Meldungen an die zentrale Datenbank sendet, sobald wieder eine Netzverbindung besteht. Der Disponent hat somit die Möglichkeit, „online“ die Arbeit der Fahrzeuge zu überwachen und, wenn nötig, sofort einzugreifen. 


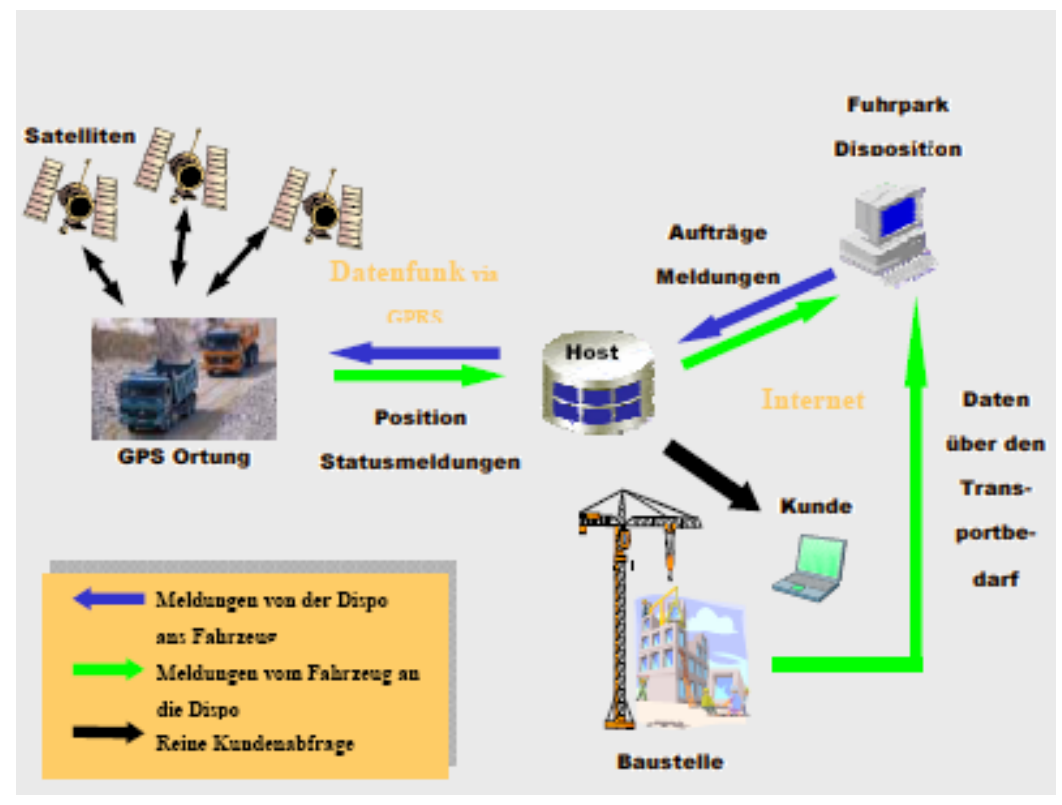

Abbildung 5: Funktionsskizze Telematiksystem

Die gesamte Kommunikation mit den Fahrzeugen wird über einen zentralen Internetserver (Host) abgewickelt, der die Daten auch für weitere Softwareapplikationen zur Verfügung stellt (siehe Abb. 5).

\section{Software}

Zur Disposition der Fahrzeuge stehen dem Nutzer zwei Softwaremodule für die Auftrags- und Fahrzeugverwaltung zur Verfügung, die ihn bei der Planung der Transportaufträge unterstützen:

- Contour Web

Kernfunktionen des Systems (Auftrags- und Stammdatenverwaltung, Kommunikation etc.)

- $\quad$ Shipping Manager

Planungsunterstützung durch graphische Anzeige der Aufträge auf die LKW auf einer Zeitachse (siehe Abb. 6)

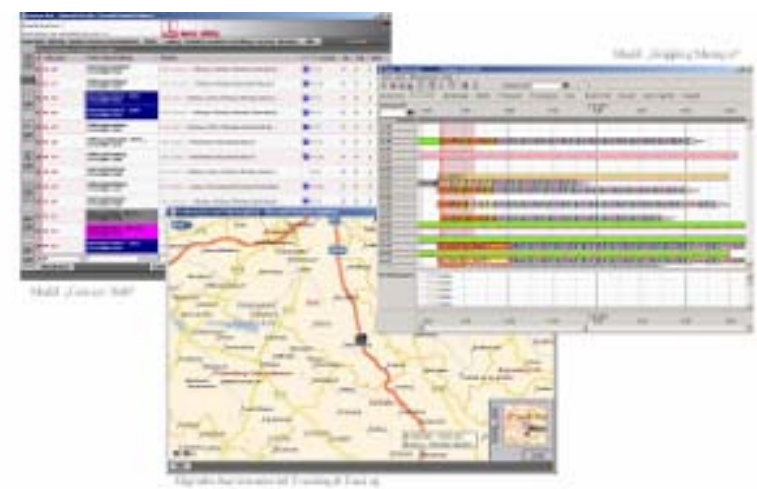

Abbildung 6: Dispositionssoftware

Die verwendete Dispositionssoftware ist internetbasiert und bietet dem Disponenten somit die Möglichkeit, seine Fahrzeuge von jedem Arbeitsplatz mit Internetzugang aus zu verwalten, ohne dass an diesem PC eine umfangreiche Installation des Programms erforderlich ist. Lediglich das Modul Shipping muss noch separat eingerichtet werden. Im Hauptmodul Contour Web werden alle Stammdaten bezüglich Fahrzeugen, Anhängern, Baustellen, Fahrern etc., die zur Auftragserstellung und -bearbeitung notwendig sind, verwaltet. Zur Gewährleistung der Datenaktualität werden neue Lieferorte bzw. Kunden über eine Schnittstelle aus SAP dynamisch aktualisiert. 
Der Disponent generiert seine Transportaufträge mit Angabe der Abhol- und Lieferadresse sowie den zugehörigen Zeiten und produktspezifischen Daten über dieses Modul. Die Zuordnung eines Auftrages auf ein Fahrzeug kann er entweder selbst durch Auswahl eines bestimmten LKW übernehmen oder alternativ automatisiert durch das Programm durchführen lassen. Über Optimierungsalgorithmen wird dann aus allen bestehenden Aufträgen und verfügbaren LKW die beste Fahrzeugauswahl für die Transportaufträge berechnet und als Vorschlag im zweiten Softwaremodul „Shipping Manager“ angezeigt. Als Kriterien für die automatische Fahrzeugauswahl werden Parameter, wie beispielsweise die Verfügbarkeit der Fahrzeuge, die Entfernung zum Abholort oder maximale Nutzlast der verfügbaren Fahrzeuge verwendet.

Der Disponent kann nun entweder den Systemvorschlag akzeptieren oder zusätzliche manuelle Änderungen der Auftragsverteilung vornehmen. Solange die Aufträge noch nicht versendet wurden, können durch einfache Drag\&Drop-Funktionalitäten die Aufträge zwischen verfügbaren Fahrzeugen sehr schnell ausgetauscht werden. Transportaufträge, die bereits versendet wurden, müssen zunächst zurückgerufen werden, um Änderungen vorzunehmen. Die Anzeige im Shipping Manager ist dynamisch und wird laufend durch die rückgemeldeten Statusdaten der Fahrzeuge aktualisiert.

Innerhalb eines frei definierbaren Zeitfensters zur aktuellen Uhrzeit werden die Aufträge, deren Abholzeitpunkt in diesem Zeitraum liegt, automatisch an die Fahrzeuge versendet.

\section{Datenerfassung in den Fahrzeugen}

Der Bordrechner in den Transportfahrzeugen erfasst alle fahrer- und auftragsbezogenen Meldungen, die für eine spätere automatisierte Abrechnung notwendig sind. Hierzu zählen beispielsweise Arbeitsbeginn- und Ende, Pausenzeiten, Nebentätigkeiten, wie Fahrzeugpflege etc. Alle Informationen eines Transportauftrags, wie Beginn des Beladevorganges Zeitpunkt der Ankunft an der Entladestelle oder Lieferscheinnummer, werden direkt digital verarbeitet und müssen nicht zusätzlich über eine manuelle Schnittstelle im EDV-System verbucht werden. Informationen stehen sofort zur Verfügung und können nahezu in Echtzeit ausgewertet werden. Neben auftragsbezogenen Daten können mit dem Bordrechner auch fahrzeugspezifische Daten, wie gefahrene Kilometer, Geschwindigkeit etc., automatisch erfasst und dokumentiert werden. Zu jeder Aktion, die im Fahrzeug generiert wird, überträgt der Bordrechner die aktuelle Uhrzeit und die GPS-Koordinaten, so dass ein durchgängiges Tracking\&Tracing (siehe Abb. 6) der Fahrzeuge möglich wird.

\section{Softwarekonzept Baustelleneinsatz}

Besonders bei der Abwicklung der internen Baustellentransporte hat sich gezeigt, dass für diesen Einsatzfall die am Markt üblichen Softwaresysteme mit ihrer Auftragslogik nicht geeignet sind. Auf Grund der hohen Anzahl an Einzelfahrten und der kurzfristigen Umplanungen auf der Baustelle, über die der Disponent im Normalfall keine Information erhält, sind die klassischen vordefinierten Aufträge mit fixen Abhol- und Lieferorten nicht geeignet. Die Fahrer und Disponenten würden bei derartiger Auftragsabwicklung nur mit zusätzlichem administrativem Aufwand belastet.

Für diesen gesonderten Einsatzfall wurde daher in Zusammenarbeit mit dem Softwarelieferanten, der Fa. ivu aus Berlin, eine spezielle Lösung entwickelt, die auf der Baustelle ein Höchstmaß an Flexibilität in der Einsatzplanung zulässt, ohne dass zusätzliche Rücksprache mit der Disposition gehalten werden muss, sobald sich die Lade- oder Entladestelle auf der Baustelle während des Tages ändert.

Grundgedanke dieses Konzeptes ist, dass über die GPS Ortung der LKW bei allen auftragsbezogenen Aktionen, wie beispielsweise Laden oder Entladen, die Geopositionen dokumentiert werden können. In Kombination mit einer vordefinierten Einteilung der gesamten Baustelle in feste Lade- und Entladestellen können diese Aktionen dann durch die Software den jeweiligen Bauabschnitten zugeordnet werden, ohne dass von außen ein Transportauftrag explizit vorgeschrieben wird. Durch Überlagerung der aktuellen GPS Position mit den vordefinierten Geopositionen innerhalb der Baustelle kann die Software die Fahrten zwischen diversen Ladeund Entladestellen nachvollziehen (siehe Abb. 7). 


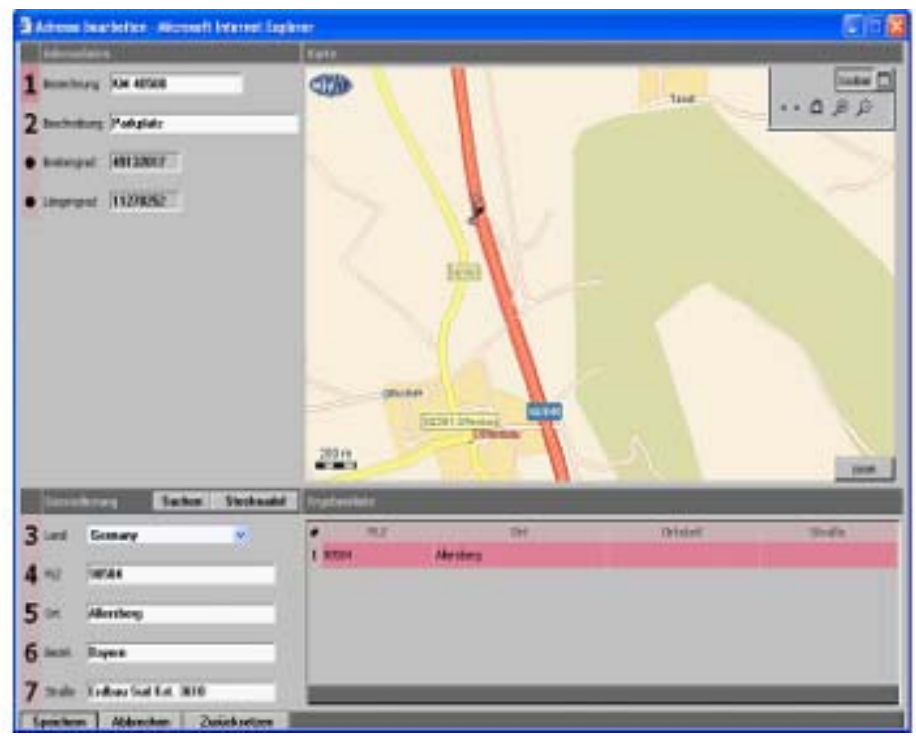

Abbildung 7: Einteilung der Baustelle in Ladestellen

Der LKW wird vom Disponenten lediglich für einen festen Zeitraum mit einer Startaufgabe einer Baustelle zugewiesen. Alle nachfolgenden Aktionen werden durch Quittierung des Fahrers am Touchscreen beim Beladevorgang am tatsächlichen Ladeort fahrzeugbezogen erfasst und an die zentrale Datenbank übermittelt. Analog erfolgt auch die Dokumentation des Entladens, indem durch den Kippvorgang automatisch eine Meldung mit der aktuellen Position und der Zeit des Vorgangs generiert wird. Mit Hilfe der Software kann somit über die kumulierten Daten die genaue Tagesleistung eines LKW auf der Baustelle sehr detailliert nachvollzogen werden.

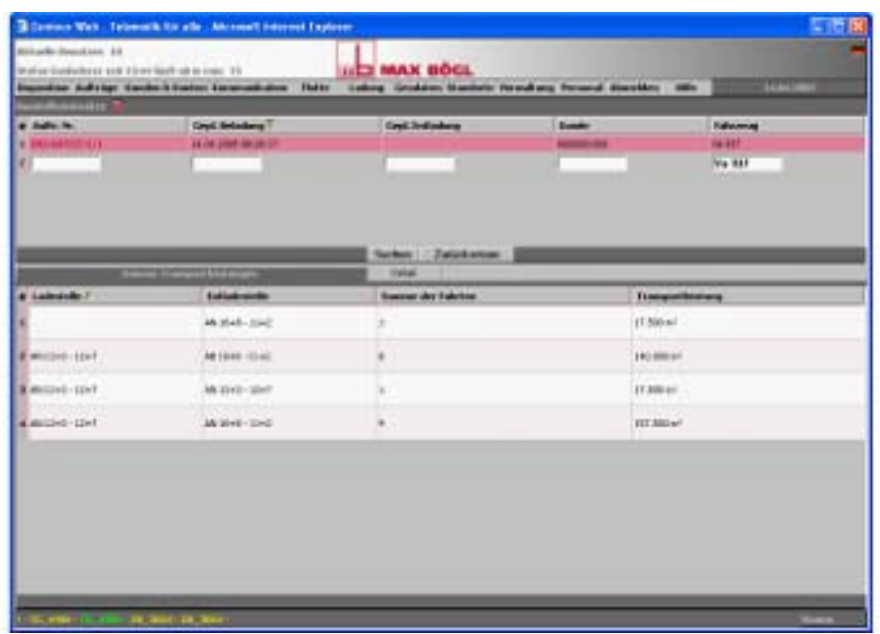

Abbildung 8: Auswertung der Leistung eines LKW

Weiterhin kann mit Hilfe dieser Leistungsinformationen tagesaktuell der Volumenfluss zwischen den verschiedenen Lade- und Entladestellen auf der Baustelle nachvollzogen werden, um eine schnelle Auskunft über den aktuellen Projektstand und die erbrachte Transportleistung zu geben (siehe Abb. 8).

\section{Dynamischer Baustellenplaner}

Auf Basis dieser zentral archivierten Daten über alle angelieferten Mengen und auch die internen Massenbewegungen kann die gesamte Leistung hinsichtlich der Transporte einer Baustelle graphisch im „Dynamischen Baustellenplaner“ dargestellt werden, um möglichst schnell einen Überblick des Baufortschritts zu vermitteln. 


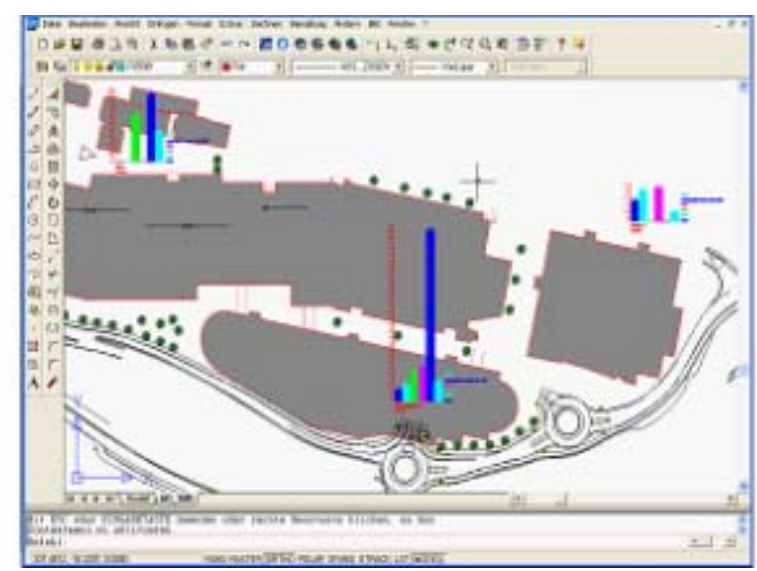

Abbildung 9: Dynamischer Baustellenplaner

Hierzu werden die 2D Baustellenpläne mit einem separaten Layer im AutoCAD ergänzt, auf dem die Planwerte aus der Kalkulation (SOLL) und die kumulierten aktuellen IST Daten aus den LKW Leistungen dargestellt werden (siehe Abb. 9). Die Darstellung der Informationen kann mittels benutzerdefinierter Abfragen hinsichtlich des Zeitraums und der Detaillierung angepasst werden. Zur übersichtlicheren Darstellung bereinigt das Tool die Zeichnung um alle nicht relevanten Informationen und blendet diese dann aus. Durch den Zeichnungseintrag kann auch für einen Betrachter, der ein Bauvorhaben nicht bis ins kleinste Detail kennt, sehr schnell der räumliche Bezug der erbrachten Leistung hergestellt werden.

\section{Einsparspotenziale durch automatisierte Datenerfassung}

Durch den Einsatz des Telematiksystem können alle Informationen eines Transportauftrags und die zugehörigen lohnrelevanten Zeiten der Fahrer direkt digital erfasst und weiter verarbeitet werden. Eine zusätzliche manuelle Verbuchung entfällt somit. Alle Fahrzeuge arbeiten mit einem einheitlichen System und liefern daher sehr gut vergleichbare Daten, da subjektive Einflüsse bei der Datenerfassung weitgehend ausgeschlossen werden. Auf Grund der erheblich detailllierteren Datenbasis ist zudem ein verbessertes Fahrzeugcontrolling möglich. Strategische Entscheidungen, wie der Einsatz von 4-Achsern oder Dumpern im Baustelleneinsatz, können durch praxisrelevante Leistungsdaten aus vergangenen Projekten gestützt werden.

Standzeiten der Fahrzeuge werden durch die verbesserte Planung und höhere Transparenz des Transportprozesses minimiert. Unnötige Nebenzeiten der Fahrer, wie beispielsweise die manuelle Leistungsdokumentation in einem LKW-Tagesbericht können entfallen. Somit wird der produktive Zeitanteil eines Fahrzeugs wesentlich erhöht und die Effektivität des gesamten Fuhrparks steigt deutlich.

Neben den direkten Einsparmöglichkeiten in der Abrechnung und Disposition der LKW, die über die eingetretene Zeiteinsparung bzw. Erhöhung der Auslastung gut monetär quantifizierbar sind, ergibt sich durch den Bordrechnereinsatz in der Zusammenarbeit mit der Baustelle noch ein zusätzlicher Mehrwert, der nur schwer finanziell zu bewerten ist. Hierzu zählen überwiegend die bessere Informationsbereitstellung für die Baustelle und die höhere Transparenz der Transportvorgänge. Die Abläufe innerhalb der Baustellen können durch die genaue Erfassung aller Fahrzeuge, die mit Bordrechnern ausgestattet sind, wesentlich besser überwacht und geregelt werden. Für die Baustelle reduziert sich somit der Aufwand für die Erstellung einer Leistungsdokumentation, da auf die Daten der Fahrzeuge zurückgegriffen werden kann. Zur Gesamtauswertung müssen diese dann lediglich noch mit Daten, die nicht bzw. noch nicht automatisch erfasst werden, kombiniert und in der gewünschten Form dargestellt werden.

\section{Ausblick}

Neue Generationen von Bordrechnern, mit höherem Leistungsumfang ermöglichen künftig auch die Abwicklung zusätzlicher Funktionen, wie die Erfassung von Lieferscheinen per Barcode. Durch zusätzliche Sensorik bzw. den Anschluss an die Fahrzeug-CAN-Busse können noch weitere 
Informationen bzgl. der LKW-Kenndaten oder auch Ladungseigenschaften erfasst werden. Ein wichtiger Punkt auf dem Weg zum papierlosen Prozess ist auch die Erfassung digitaler Unterschriften zur Leistungsquitterung, die mit neuer Hardware möglich wird.

Der LKW repräsentiert lediglich einen Teil im Arbeitsprozess auf der Baustelle. Damit ein durchgängiges, automatisiertes Controlling des Gesamtprozesses vom Bagger über den LKW bis hin zu Raupe und Walze möglich wird, muss in einem nächsten Schritt die Datenerfassung an der Baumaschine mit in das Konzept einbezogen werden. Durch die Einbindung aller Maschinenleistungs- und -qualitätsdaten, wird der nächste wichtige Schritt in Richtung „Gläserne Baustelle“ gemacht. Den Bauleitern wird somit die Möglichkeit gegeben, ihre Projekte noch besser zu planen und zu überwachen. Weiterhin können die Baumaschinen durch die Überwachung der internen Prozessdaten, wie beispielsweise Hydraulikdrücke, Verbräuche, Betriebszeiten sowie Voll- und Leerlastbereichen, materialschonender und somit auch kostengünstiger eingesetzt werden.

Im weiteren Projektverlauf ist angedacht, den Dynamischen Baustellenplaner vom 2D ins 3D zu übertragen und zusätzliche Informationen zu integrieren, da viele Zusammenhänge durch den räumlichen Eindruck noch leichter vermittelt werden können. Zusätzlich soll dieses Tool in Kombination mit der Baumaschinen-Gerätedatenbank EIS (bitte auflösen), die bereits im Vorfeld am Lehrstuhl fml entwickelt wurde, auch Einsatz in der Planung der Baustellen finden, um auch in diesem Bereich ein objektives Hilfsmittel zur Verfügung zu stellen. Der Bauablauf lässt sich im Anschluss durch die Auswahl eines Maschinenparks aus der Datenbank EIS und die gegebene Massenverteilung aus den Vermessungsdaten vorab simulieren.

\section{Fazit}

Telematiksysteme bieten auch für den Baustelleneinsatz einen sehr hohen Nutzen, wenn sie konsequent in einem durchgängigen Konzept eingesetzt werden, das auf die individuellen Bedürfnisse der Anwender zugeschnitten ist. Durch die automatisierte Auftragsverwaltung können weit reichende Einsparpotenziale genutzt werden, die sich nicht nur auf die Transportabteilungen im Unternehmen beschränken. Neben der Fahrzeugdisposition ergeben sich durch den Bordrechnereinsatz für Baufirmen noch eine Vielzahl weiterer Nutzungsmöglichkeiten, wie beispielsweise die Verfolgung von Massenströmen auf den Baustellen oder die Aufzeichnung von qualitätsrelevanten Daten, wie Transportdauer oder -temperatur, die vom Benutzer je nach Anforderung automatisch dokumentiert werden können. Damit können behördlich geforderte Qualitätsmerkmale problemlos dokumentiert und später bei Bedarf nachgewiesen werden.

Baufirmen können sich durch den Einsatz von Telematiksystemen zur Verbesserung des gesamten Prozessablaufs bereits heute sehr gut für die Aufgaben von morgen rüsten. 